\title{
First Demonstration of a Low Loss 37-cell Hollow Core Photonic Bandgap Fiber and its Use for Data Transmission
}

\author{
N. K. Baddela, M. N. Petrovich, Y. Jung, J. R. Hayes, N.V. Wheeler, D. R. Gray, N. Wong, F. Parmigiani, \\ E. Numkam, J. P. Wooler, F. Poletti, D. J. Richardson \\ Optoelectronics Research Centre, University of Southampton, Highfield Campus, SO17 1BJ, Southampton, UK \\ nkb1c10@orc.soton.ac.uk
}

\begin{abstract}
A low loss $(4.5 \mathrm{~dB} / \mathrm{km}) 37$-cell core $\mathrm{HC}-\mathrm{PBGF}$ is reported for the first time. Detailed modal analysis using time of flight and $\mathrm{S}^{2}$ techniques, and error-free 40Gbit/s single mode data transmission are presented.

OCIS codes: (060.5295) Photonic crystal fibers; (060.2330) Fiber optics communications
\end{abstract}

\section{Introduction}

Hollow core photonic bandgap fibers (HC-PBGFs) are emerging as credible candidates for data transmission applications because of their low nonlinearity, low latency and predicted ultralow loss. Recently, 19-cell HC-PBGFs with wide bandwidths $(>150 \mathrm{~nm})$ and a loss of a few $\mathrm{dB} / \mathrm{km}$ have been demonstrated [1]. Their ability to support high capacity data transmission at $1.55 \mu \mathrm{m}$ and $2 \mu \mathrm{m}$ has also been established [2,3]. However, for HC-PBGFs to stand a realistic chance of competing with current transmission fibers, further loss reduction needs to be achieved whilst maintaining a wide transmission bandwidth. Since the fundamental loss limit of HC-PBGFs is determined by surface roughness scattering [4], one possible route to loss reduction is to increase the core size, e.g. from 19-cell to 37-cell, thus reducing the amount of overlap between optical field and glass. In practice, however, the fabrication of HC-PBGFs with bigger core surrounds presents a number of challenges, including the presence of potentially more surface modes as compared to 7 and 19-cell HC-PBGFs [5], and a substantial increase in the number of core guided modes, to the point that a 37-cell HC-PBGF was previously expected to have little practical value [4].

In this work, we report fabrication of the first ever 37-cell (37c) HC-PBGF. The fiber has a low minimum loss of $4.5 \mathrm{~dB} / \mathrm{km}$ at $1550 \mathrm{~nm}$ and a wide $3-\mathrm{dB}$ bandwidth of $85 \mathrm{~nm}$, obtained by applying similar core surround engineering as previously described [1]. Modal characterization using Time of Flight and $S^{2}$ techniques, shows that, remarkably, the levels of crosstalk between lower order mode groups is low and comparable to that previously measured in 19 cell fibers, and thus also this 37c HC-PBGF can be operated as effectively single moded. As a proof of that we report $40 \mathrm{Gbit} / \mathrm{s}$ error-free single mode data transmission over $>300 \mathrm{~m}$ of fiber.

\section{Fiber Fabrication and Characterization}

The 37 cell HC-PBGF was fabricated using the conventional dual-step stack and draw technique. The fiber has $61 / 2$ rings of cladding holes and 37 capillaries were removed from the center of the array to form the core. To reduce the impact of surface modes inside the bandgap region, no central core tube was used [3]. The fiber has an average pitch, relative hole size and core diameter of $4.4 \mu \mathrm{m}, 0.97$ and $37 \mu \mathrm{m}$, respectively (Fig 1a). The spectral attenuation was measured via a cutback method using a white light source and optical spectrum analyzer. A large mode area holey fiber (LMA 25 from NKT Photonics A/S) was used to couple light into the HC-PBGF ensuring good match to the mode field diameter of the fundamental mode $\left(\mathrm{LP}_{01}\right)$. Transmission plots and loss data obtained from a careful cutback from $320 \mathrm{~m}$ to $10 \mathrm{~m}$ are shown in Fig. 1(b,c). The fiber has a minimum loss of $4.5 \mathrm{~dB} / \mathrm{km}$ at $1550 \mathrm{~nm}$. Only two groups of surface modes are observed within the bandgap, and by optimizing the relative expansion of core and cladding a 3-dB transmission bandwidth of $85 \mathrm{~nm}$ was obtained, with significant potential for further optimization.
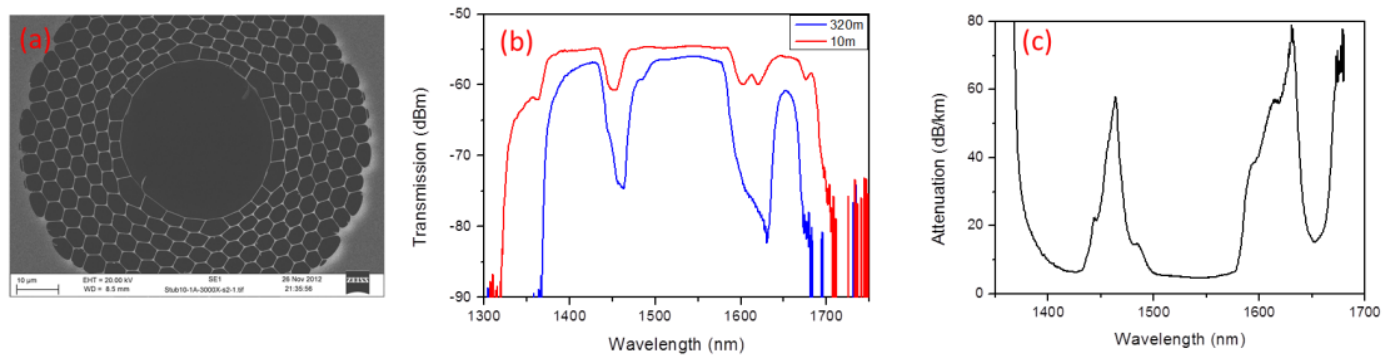

Fig. 1. (a) Scanning electron micrograph of the 37 cell HC-PBGF, (b) White light transmission through 320m (blue) and $10 \mathrm{~m}$ (red) of $37 \mathrm{cell} \mathrm{HC}$ PBGF and (c) Spectral attenuation measured by cutback (320m to $10 \mathrm{~m})$ using white light source 
Theoretical models indicate a sharp increase in the number of core modes as the core diameter is enlarged. An ideal $37 \mathrm{c}$ HC-PBGF structure is predicted to support as many as 80 modes, including degeneracies. To gain insight into the modal behavior of these novel fibers, we have mapped the modal content via an $\mathrm{S}^{2}$ measurement and observed propagation using a Time-of-Flight $(\mathrm{ToF})$ technique. The results of the $S^{2}$ measurement $(10 \mathrm{~m}$ fiber length) are shown in Fig 2(a). The differential group delay (DGD) of the $\mathrm{LP}_{11}, \mathrm{LP}_{12}, \mathrm{LP}_{21}$ and $\mathrm{LP}_{02}$ modes was found to be $\sim 4-6$, 7-8, 9-11 and 12-14 ps/m, respectively, roughly 50\% lower than in a 19c- HC-PBGF, resulting from the larger core and as expected from simulations [6]. Interestingly however, fewer modes are observed than expected in an ideal structure, with an apparent cut-off after the $\mathrm{LP}_{02}$ mode that requires further investigation. The ToF measurement was carried out by using sub-ps pulses from a mode-locked laser and a $10 \mathrm{GHz}$ bandwidth sampling oscilloscope. The results for a $310 \mathrm{~m}$ length of fiber under $\mathrm{LP}_{01}$-optimised launch conditions are shown in Fig.2(b). The large extinction ratio of $\sim 23 \mathrm{~dB}$ with which all high order modes can be suppressed and the low level of distributed cross talk observed in our measurements, indicate the potential of using this fiber for data transmission in an effectively single mode regime.
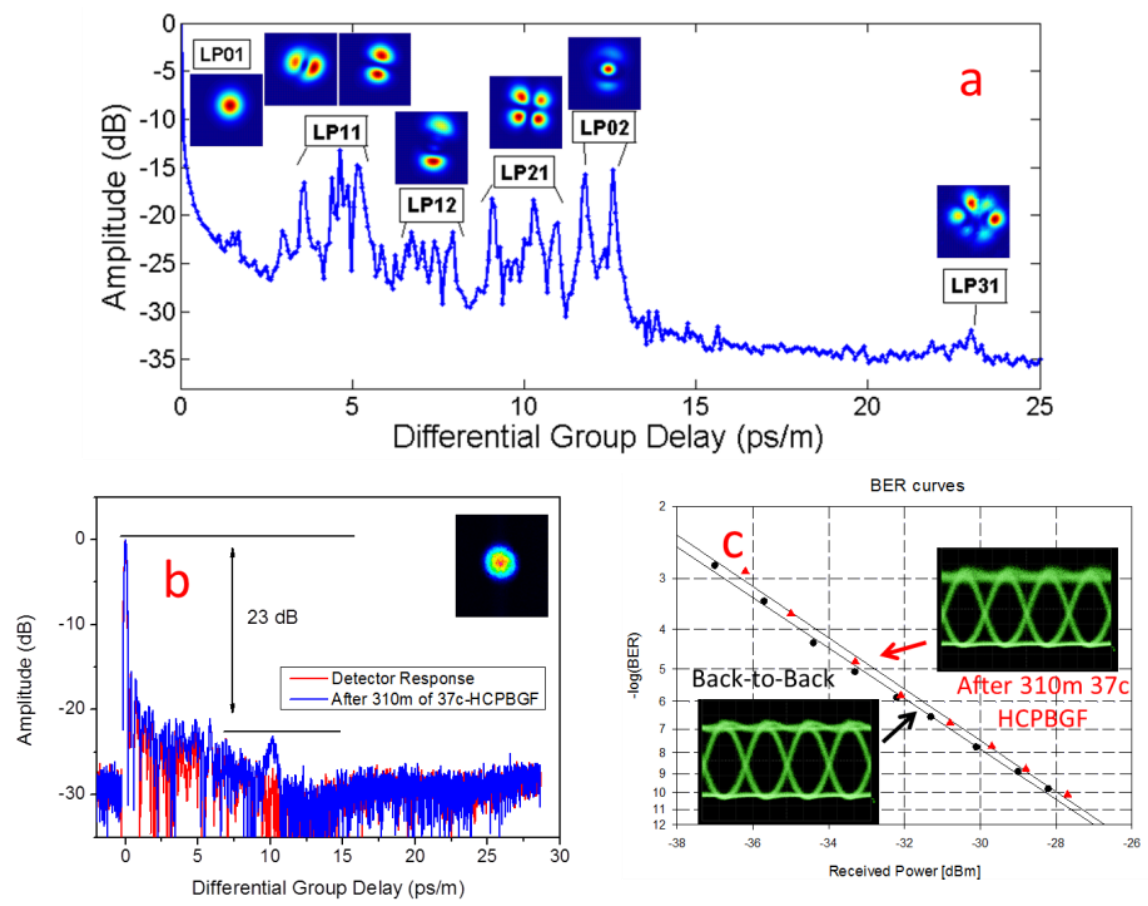

Fig 2 (a) S ${ }^{2}$ Modal characterization, (b) ToF measurements showing selective mode excitation, (c) $40 \mathrm{Gbit} / \mathrm{s}$ single mode OOK data transmission.

To confirm this, we have transmitted on-off keying modulated $40 \mathrm{Gbit} / \mathrm{s}$ data streams through $310 \mathrm{~m}$ of this $37 \mathrm{c} \mathrm{HC}$ PBGF. Error-free transmission with sub-dB penalty as compared to back-to-back measurements, using a preamplified optical receiver and very clean and open eye diagrams were observed (Fig. 2 (c)). It seems realistic that with a suitable mode multiplexing scheme this fiber could be exploited for mode group division multiplexing to achieve high capacity data transmission. We also believe that by further structural and process optimisation there is a realistic prospect of further reducing losses and widening the bandwidth of this fiber type.

This work was supported by the EU 7th Framework Programme under grant agreement 228033 (MODE-GAP) and by the UK EPSRC through grants EP/I01196X/1 (HYPERHIGHWAY) and EP/H02607X/1.

\section{References}

[1] N. V. Wheeler et al., "Wide-bandwidth, low-loss, 19-cell hollow core photonic band gap fiber and its potential for low latency data transmission," OFC 2012, PDP5A.2.

[2] R. Slavik et al., "1.45 Tbit/s low latency data transmission through 19-cell hollow core photonic band gap fibre,” ECOC 2012 , Mo. 2. F. 2.

[3] M. N. Petrovich et al., "First demonstration of 2 micron data transmission in a low-loss hollow core photonic bandgap fiber" ECOC 2012, Th 3.A.5(PDP).

[4] P. J. Roberts, et al., "Ultimate low loss of hollow-core photonic crystal fibres," Optics Express, vol. 13, pp. 236-244, Jan 10 2005.

[5] M. Digonnet, et al., "Simple geometric criterion to predict the existence of surface modes in air-core photonic-bandgap fibers," Opt. Express 12, 1864-1872 (2004).

[6] D. R. Gray et al., "Complementary analysis of modal content and properties in a 19-cell hollow core photonic band gap fiber using time-of flight and $\mathrm{S}^{2}$ techniques", ECOC 2012 Mo. 2. F.1. 